\title{
Simulation on large scale of acoustic signals for array processing
}

\author{
Gerard Llort-Pujol, Christophe Sintes, Thierry Chonavel, Didier Guériot and René Garello \\ Institut TELECOM - TELECOM Bretagne, CNRS Lab-STICC UMR 3192 \\ Technopôle Brest Iroise, CS 83818 - 29238 Brest, France \\ Email: \{gerard.llort, christophe.sintes, thierry.chonavel, didier.gueriot,rene.garello\}@ telecom-bretagne.eu
}

\begin{abstract}
Due to operational constraints for underwater data acquisition, simulating realistic sonar data, like images, swath bathymetry profiles or interferometric signals, is crucial for tuning detection and classification algorithms according to sensors settings, sea-bottom nature and topography. Moreover, the robustness of any performance estimation or prediction can be greatly enhanced, as soon as such a simulation tool provides a modular and flexible underwater world representation (multiple sensors, environments and acquisition conditions).

For signal and array processing, it is essential not only to generate the signal energy backscattered by a resolution cell, but also to produce a phase information that conveys its theoretical statistical properties. To this end, this paper proposes a Brownian motion-based approach to generate complex Gaussian signals from the contribution of a set of extended single scatterers inside a resolution cell. The resulting process preserves the conservation of energy when integrating on surfaces, as well as the decorrelation between different areas of the sea bottom, and the right interference between two sensors for interferometric applications.
\end{abstract}

\section{INTRODUCTION}

Simulating realistic sonar data, such as images, swath bathymetry profiles or interferometric signals, is becoming a key tool for reducing manufacturer's cost of data acquisitions. Thus, simulators permitting a modular and flexible underwater world representation try to replace sea surveys, necessary to evaluate performances of detection and classification algorithms.

Traditional simulators are often based on ray tracing and aggregation [1]. With such an approach, the energy of the sonar image, after aggregation, is directly linked to the density of rays intersecting a specific area of the described scene. It then supposes to use enough rays to reach a proper statistical behavior. The lack of rays leads to misrepresentation of involved phenomena. In [2], we presented a simulator that still relies on the use of acoustic rays but adds a volume description of the propagation by building tubes. For this work, the simulator describes the scene by using patches of energy contained in elementary tubes delimited by four rays. This technique allows a global and exact description of the contributions of the volumes on receiving sensors and drastically reduces the number of launched rays. A full description of this simulator is supplied in [2].

The underlying key idea for this simulator is to be parametric and adaptive. These goals are achieved by storing the full propagation history of all the transmitted tubes, each tube being related to an elementary contribution. This approach is very interesting because it makes it possible to preserve all the details that can play a role in the data output sensor simulation as the construction of such simulated output becomes only a matter of postprocessing. For instance, in a simulation where a vehicle supports two sensors, say one multibeam echosounders and one sidescan sonar, the acoustic propagation history is the same; only the aggregation part remains specific to each sensor.

The energy coming back from the sea bottom is coherent and the level of this energy results from a very intricate combination of several contributing parts of the resolution cell as a resolution cell corresponds to an elementary temporal footprint of the sonar after demodulation, on sea bottom. Many works [3] [4] have tried to represent properties of these resolution cells, such as continuous and exact model for coherent resolution cell based on either rough surface theory or micro roughness approach, or phenomenological approaches based on discrete interference. The difficulty remains the generation of coherent sonar signals with a realistic phase information that matches the right statistics, thus allowing array processing. To evaluate the correct simulation of the phase part, interferometry is used to interfere signals, leading to a first step towards array simulation.

If we want to make interfere signals coming from the sea bottom, each scatterer should be coherent with itself, but not with adjacent scatterers. A discrete simulation can directly be performed but obviously, not a continuous one. To overcome this difficulty, in [5], we proposed a new approach to generate complex signals, that consists in dividing the sea bottom into elementary blocks or extended single scatterers with specific properties. This continuous splitting preserves both the conservation of energy and the decorrelation between different areas of the sea bottom. However, these extended scatterers do not have a proper identity so that the same scatterer seen from different angles is not the same and, therefore, cannot be identified.

Thus, the goal of this paper is to propose a signal-generation approach that assigns a predefined identity to each single scatterers so that it backscatters the same information for different tracks. The solution is based on a Brownian motion process that permits the generation of two-dimensional uncorrelated complex Gaussian signals. This way, the conservation of energy is preserved, signals coming from different areas 


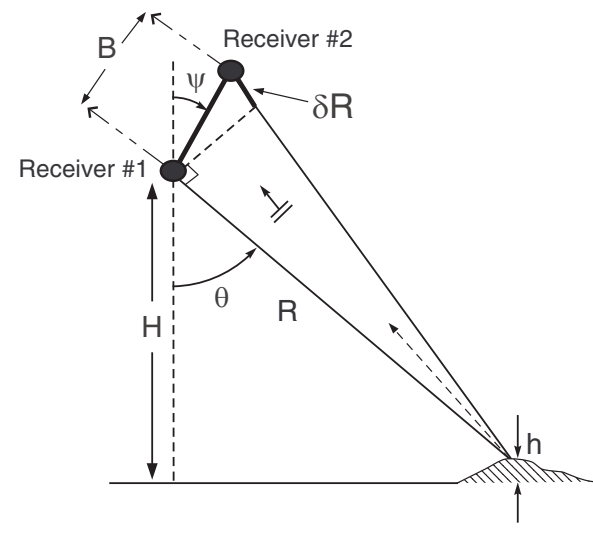

Fig. 1. Sonar interferometry geometry, defining the baseline $B$, propagationpath delay $\delta R$ between sensors, arrival angle $\theta$, steering angle $\psi$, and sonar altitude $H$.

of the sea bottom are uncorrelated, and finally, the generated phase can be used in interferometry applications.

\section{Simulating INTERFEROMETRIC SONAR SIGNALS}

\section{A. Context}

Modern bathymetry applications in sonar [6] [7] aim at estimating the two-way propagation time and the arrival angle of an emitted signal backscattered by the seafloor in order to determine the sea-bottom depth. Thus, a digital terrain model is obtained providing a three-dimensional mapping of a given surface.

Interferometry [8] [9] is a widely-used method that provides an accurate bathymetric information by measuring the phase difference between two sensors. The main interest of interferometry is to perform bathymetry at grazing angles in order to increase the swath. The principle of interferometric bathymetry is based upon the spacing between two close receivers causing a propagation-path difference $\delta R$ on the reception. The related phase difference $\Delta \varphi$ between the two receivers, recorded at time-sampling rate, can be geometrically obtained from Fig. 1 :

$$
\Delta \varphi=\frac{2 \pi}{\lambda} B \cos (\theta+\psi)+2 \pi m
$$

where $\theta$ stands for the direction of arrival of the backscattered signal, and $\psi$ denotes the steering angle, $\lambda$ is the acoustic wavelength, and $B$ is the inter-receiver spacing, commonly called baseline. As the phase difference is measured $2 \pi$ modulo [8], the parameter $m$ is introduced into the interferometry equation as a phase rotation counter to remove the phase ambiguities [10]. It is important to note that (1) is valid only under non dispersive medium hypothesis.

\section{B. Extended single scatterers}

A classical phenomenological approach [11] [12] to simulate backscattered discrete signals is based on a simple and atomistic model:

$$
F=\sum_{i=1}^{N} a_{i} e^{j \phi_{i}} s(t)
$$

where $F$ represents the coherent summation of the signal transmitted back by the $N$ single scatterers belonging to a resolution cell. Parameters $a_{i}$ and $\phi_{i}$ represent the amplitude and phase from the single scatterer $i \in[1, N]$, while $s(t)$ gives the signal initially transmitted by the sonar (which is the incident signal). This model is very easy to understand: each scatterer belonging to the resolution cell receives the incident signal and transmits it back with modified power and phase, depending on the scatterer properties. Within a resolution cell, the number $N$ of scatterers, amplitude $a_{i}$ and phase $\phi_{i}$ of every scatterer can be random.

The main difficulty with this approach is to define the basic single scatterer properties fitting both experimental and theoretical models. Moreover, the scatterer spatial properties are another very crucial point to consider and especially the cross-correlation function between scatterers. Indeed, as each sensor observes the sea bottom from a different point of view, both footprint shift and baseline decorrelation [13] [14] are fundamental to reach a realistic simulation

In [5], we showed that the contribution of the resolution cell to the generation of a complex signal could be continuously modeled through the introduction of the notion of extended single scatterers. Indeed, instead of generating complex signals by a simple aggregation of punctual scatterers as in (2), we proposed the integration of the contributions of single scatterers inside a resolution cell along the sampling time. Thus, the complex signal contribution $z(k+1)$ received at sampling time $k+1$ can be written, in a continuous form, as the integration of any single scatterer contained in a resolution cell between sampling times $t_{\min }$ and $t_{\max }$ :

$$
\begin{aligned}
z(k+1) & =\int_{t_{\min }}^{t_{\max }} \mathbb{1}_{\left[0, T_{s}\right]}\left((k+1) T_{s}-u\right) e^{-j 2 \pi f u} d u(3) \\
& =e^{j 2 \pi f \frac{t_{\max }+t_{\min }}{2}} \cdot \frac{\sin \left(\pi f\left(t_{\max }-t_{\min }\right)\right)}{\pi f}(4)
\end{aligned}
$$

where $\mathbb{1}_{A}(u)$ stands for the indicator function and $T_{s}$ denotes the sampling period. Observing the solution of the integral, the resulting signal phase is parameterized by the phase center of the resolution cell, while the sinc function depends on the time duration of the resolution cell, i.e. the sampling period. We can see then that the signals obtained from different resolution cells have the same form, and therefore, the resulting phase interference is zero. In other words, with this modeling, resolution cells have no phase signature helping distinguish them.

To overcome this problem, the sea bottom is divided into elementary blocks or extended single scatterers with specific properties. Thus, based on the linearity of the integral (3), a Chasles relation can be derived for adjacent elementary blocks with the same properties. Thus, the contribution of $N$ extended scatterers inside a resolution cell to the generation of the complex signal $z(k+1)$ is evaluated as:

$$
z(k+1)=\sum_{n=1}^{N} e^{j 2 \pi f \frac{t_{b, n}+t_{a, n}}{2}} \cdot \frac{\sin \left(\pi f\left(t_{b, n}-t_{a, n}\right)\right)}{\pi f} \cdot e^{j \phi_{n}}
$$


where $t_{a, n}$ and $t_{b, n}$ stand for the temporal limits of a given extended scatterer $n$, and $\phi_{n}$ is a uniform random variable that provide a unique identity to the extended scatterer.

This approach has the two main drawbacks: the first one concerns the statistical properties of the generated signals because they do not correspond to actual interferometry phase statistics [15]. The second drawback deals with the identity assigned to each extended scatterer: although this solution yields the good interference [5], a given scatterer belonging to a seafloor mapping cannot be identified if a second survey ensonifies the same resolution cell because the identity is not related to the scatterer position. Thus, the improvement of this modeling consists in assigning a predefined (phase) identity to each extended scatterer, related to its position. This georeferenced identity can be used by geographic information systems (or other cartographic methods) to identify the same position each time it is ensonified by different surveys.

Analyzing the problem in two dimensions, the idea is to create a map where each position (or pixel) is a random variable, holding phase statistics in terms of decorrelation. Different approaches were regarded, such as Perlin (gradient) noise [16], used in computer texture graphic technology, or fractional Brownian motion [17], conveying Gaussian properties. The great advantage of the Perlin noise is that it is possible to compute the value of a given pixel independently of its neighborhood, but the generated signal has no real statistical sense. Conversely, a Brownian-based solution does permit the generation of uncorrelated Gaussian signals. Thus, the next section discusses the Brownian-based solution and how it is adapted to generate both amplitude and phase information. Interferometry is then used to verify the right signal simulation.

\section{BROWNIAN-BASED SIGNAL SIMULATION}

\section{A. Brownian derivation}

The integral (3) can be written in a general form as:

$$
z(k+1)=\int_{t_{\min }}^{t_{\max }} g(u) \cdot e^{-j 2 \pi f u} d u
$$

where the function $g(u)$ represents the random signal associated to extended scatterers. This function may be unknown, but the solution of this integral for each interferometer receiver can be written as:

$$
\begin{aligned}
& z_{1}(k+1)=H\left(t_{b}\right)-H\left(t_{a}\right) \\
& z_{2}(k+1)=H\left(t_{d}\right)-H\left(t_{c}\right)
\end{aligned}
$$

where $H\left(t_{x}\right)$ denotes the antiderivative or cumulative function of (6) at $t_{x}$,

$$
H\left(t_{x}\right)=\int_{-\infty}^{t_{x}} g(u) \cdot e^{-j 2 \pi f u} d u
$$

and $t_{c}\left(t_{b}\right)$ and $t_{a}\left(t_{z}\right)$ are the temporal upper and lower boundaries of the resolution cell associated to the receiver \#2 (receiver \#1), respectively. An illustrative sketch is presented in Fig. 2.

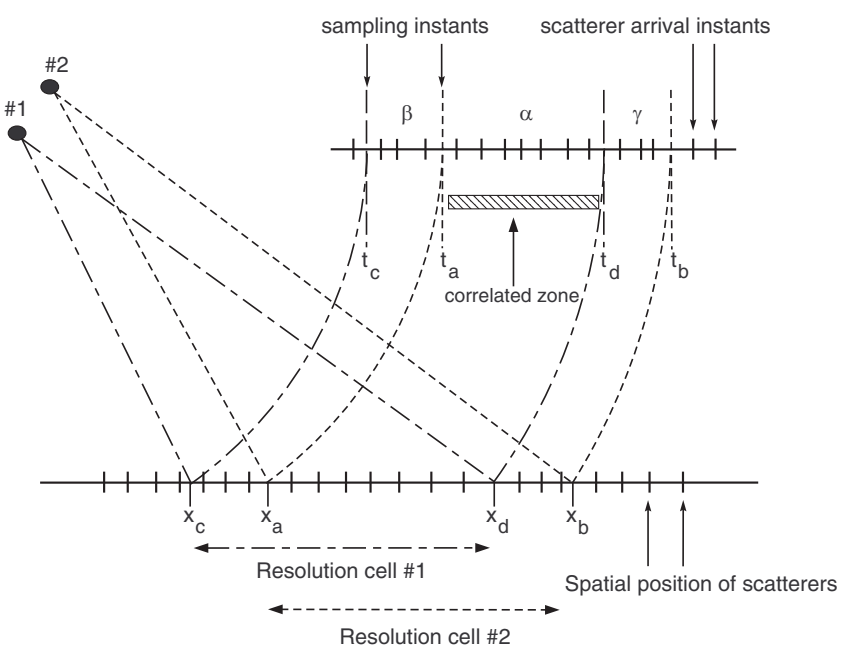

Fig. 2. Split zone of integration due to the decorrelation between receivers.

In order to model $H(x)$, let us evaluate the cross-correlation function between the received signals:

$$
\begin{aligned}
z_{1}(k+1) z_{2}^{*}(k+1)= & {\left[H\left(t_{d}\right)-H\left(t_{c}\right)\right]\left[H\left(t_{b}\right)-H\left(t_{a}\right)\right] } \\
= & {[\underbrace{H\left(t_{d}\right)-H\left(t_{a}\right)}+\underbrace{H\left(t_{a}\right)-H\left(t_{c}\right)}] } \\
& \times(\underbrace{H\left(t_{b}\right)-H\left(t_{d}\right)}+\underbrace{H\left(t_{d}\right)-H\left(t_{a}\right)}] \\
= & \alpha^{2}+\alpha \gamma+\beta \alpha+\beta \gamma
\end{aligned}
$$

with $\alpha, \beta$ and $\gamma$ being defined in Fig. 2 as the increments between antiderivatives, or the correlated $(\alpha)$ or uncorrelated ( $\beta$ and $\gamma$ ) contributions. Equation (10) permits the modeling of the received signals. Indeed, in order to ensure a correct cross-correlation between signals and independent increments, the following relation must be held:

$$
\begin{aligned}
\mathbb{E}\left\{z_{1}(k+1) z_{2}^{*}(k+1)\right\} & =\mathbb{E}\left\{\alpha^{2}\right\} \\
\Rightarrow \mathbb{E}\{\alpha \gamma\} & =0 \\
\Rightarrow \mathbb{E}\{\alpha \beta\} & =0 \\
\Rightarrow \mathbb{E}\{\beta \gamma\} & =0
\end{aligned}
$$

Now, if signal $g(u)$ associated to extended scatterers is assumed uncorrelated white Gaussian distributed, its integration in (8) leads the antiderivatives $H\left(t_{x}\right)$ to be a Wiener process, also known as Brownian motion, as pointed out in [18]. As a result, the increments given in (7) are also Gaussian distributed, producing Gaussian signals at reception.

Therefore, instead of assigning a uniform random phase to each extended scatterer as in (4), the new modeling regards the signals associated to each extended scatterer as zeromean complex Gaussian noises $W(t)$, involving correlation and covariance [17] [18] between two antiderivatives, given 


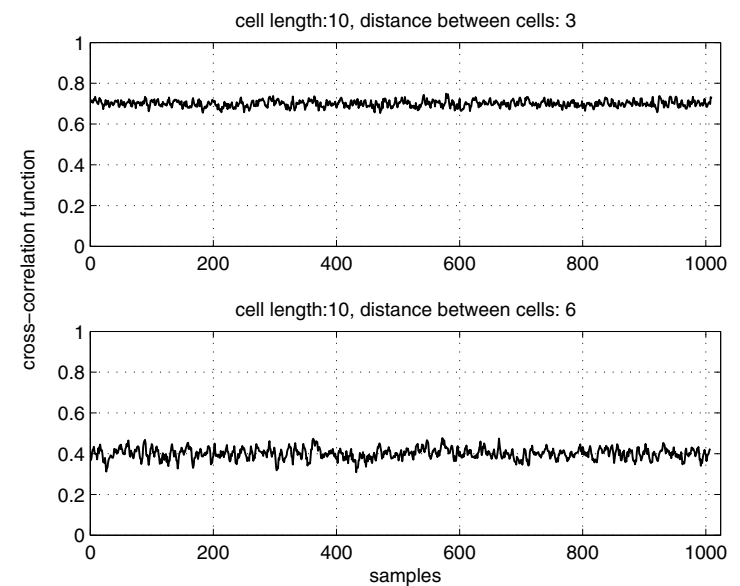

Fig. 3. One-dimensional cross-correlation function between two resolution cells associated to the cell positioning shown in (2). Upper plot: $t_{b}-t_{a}=t_{d}-t_{c}=10$ and $t_{a}-t_{c}=3$. Lower plot: $t_{b}-t_{a}=t_{d}-t_{c}=10$ and $t_{a}-t_{c}=6$.

by:

$$
\begin{aligned}
\operatorname{cov}\left(H\left(t_{1}\right), W\left(t_{2}\right)\right) & =\min \left(t_{1}, t_{2}\right) \\
\operatorname{corr}\left(H\left(t_{1}\right), W\left(t_{2}\right)\right) & =\frac{\min \left(t_{1}, t_{2}\right)}{\sqrt{t_{1} t_{2}}}
\end{aligned}
$$

These features above are supplied for the one-dimensional case. However, the simulator works with ray tubes that, projected on a cartographic map, results in two-dimensional footprints. In order to obtain the same statistical characteristics as in (11) in the two-dimensional case, i.e. independent Gaussian increments and right cross correlation, we used the two-parameter Brownian motion process, $\{W(s, t), s, t>0\}$, also known as the Brownian sheet [19]. Thus, the Brownian sheet $W(s, t)$ has also independent increments, in the sense that for all disjoint rectangles $R_{1}, R_{2}, \ldots$, in $\mathbb{R}_{+}^{2}, W\left(R_{1}\right)$, $W\left(R_{2}\right), \ldots$, are independent.

\section{B. Brownian simulation}

A 1-D Brownian motion can be easily simulated by creating a zero-mean Gaussian random vector and computing the cumulative sum function. In order to verify the right properties of cross correlation between resolution cells, a 1024-length Brownian vector composed of 1024 observations was created. Then, the cross-correlation function was computed between two resolution cells of ten-unit length, obtaining plots in Fig. 3. For the upper plot, the resolution cells were three units apart, and for the lower plot, six units apart (see cell distributions in Fig. 2). The resulting correlations were 0.7 and 0.4 (on average), respectively, as expected.

In the two-dimensional case, the simulation of a Brownian sheet is carried out by creating a zero-mean Gaussian random matrix, applying the cumulative sum function along one axis direction and then, applying this function again to the result along the other axis direction. We checked the correct crosscorrelation between 2-D resolution cells: Fig. 5 displays the correlation between two quadrilaterals, 10-unit length, 2 units

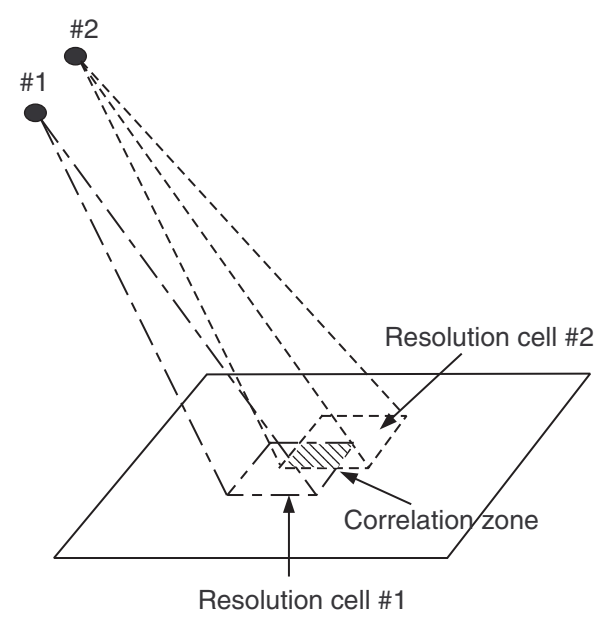

Fig. 4. Split zone of integration due to the decorrelation between receivers for a 2-D mapping.

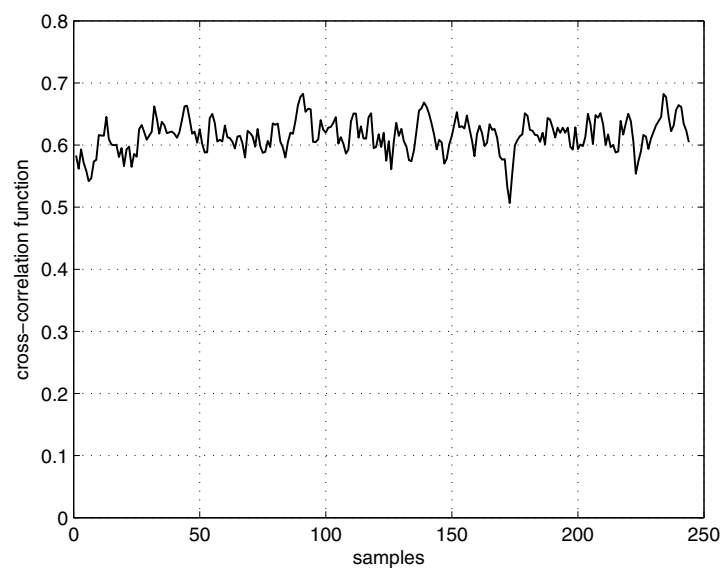

Fig. 5. Two-dimensional cross-correlation function between two quadrilateral resolution cells, two-unit length, two-units apart, arranged as shown in Fig. 4.

apart for each side (see illustrative sketch in Fig. 4). As expected, the cross-correlation for different observations is (roughly) $0.64(0.8 \times 0.8)$.

Note that the precalculation of the map demands the computation of the geographic location of each extended scatterer, which, in turn, depends on sonar parameters, such as the receiver position or the inter-receiver spacing (baseline). To simplify the precalculation, one solution is to make the integral (6) independent on the position of the sonar receivers when precalculating the map. This is obtained by a series expansion of the integral (6).

\section{Series expansion approach}

The integration (6) corresponding to the contribution of the extended scatterers to the received signal $z(k+1)$ can be rewritten as a function of the spatial coordinates instead of 


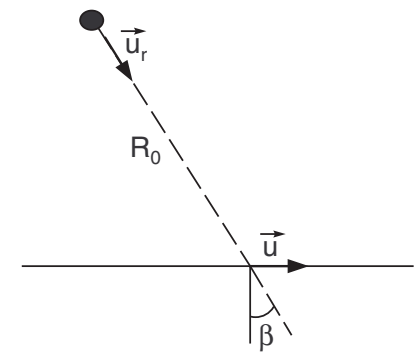

Fig. 6. Two-dimensional cross-correlation function between two quadrilateral resolution cells arranged as shown in Fig. 4.

the sampling time:

$$
z(k+1)=\int_{x_{\min }}^{x_{\max }} g(v) \cdot e^{-j 2 \pi \frac{r(v)}{\lambda}} d v
$$

with $x_{\max }$ and $x_{\min }$ defining the projection of spatial resolution-cell footprint on the seafloor. The two-way propagation path $r(v)$ can be expressed as a spatial vector $\vec{r}$ as illustrated in Fig. 6.

$$
\vec{r}=\overrightarrow{R_{0}}+\vec{v}
$$

whose Euclidian norm can be determined as:

$$
\begin{aligned}
\|r\|^{2} & =\left\|R_{0}\right\|^{2}+\|v\|^{2}+2 \overrightarrow{R_{0}} \vec{v} \\
& =\left\|R_{0}\right\|^{2}\left(1+\frac{\|v\|^{2}+2 \overrightarrow{R_{0}} \vec{v}}{R_{0}}\right)
\end{aligned}
$$

This norm can be simplified by applying a Taylor series expansion with respect to $R_{0}^{1}$ :

$$
\begin{aligned}
\|r\| & \simeq R_{0}+\frac{v^{2}+2 \overrightarrow{R_{0}} \vec{v}}{2 R_{0}} \\
& \approx R_{0}+\frac{\overrightarrow{R_{0}} \vec{v}}{R_{0}} \\
& =R_{0}+\frac{R_{0} \overrightarrow{v_{r}} \vec{v}}{R_{0}} \\
& =R_{0}+v \sin \beta
\end{aligned}
$$

where $\beta$ is the measure of the angle between $\overrightarrow{v_{r}}$ and $\vec{v}$ as shown in Fig. 6. As a result, the propagation distance $r(v)$ in (14) can be replaced by (17d), yielding:

$$
\begin{aligned}
z(k+1) & =\int_{x_{\min }}^{x_{\max }} g(v) \cdot e^{-j 2 \pi \frac{R_{0}+v \sin \beta}{\lambda}} d v \\
& =e^{-j 2 \pi \frac{R_{0}}{\lambda}} \int_{x_{\min }}^{x_{\max }} g(v) \cdot e^{-j 2 \pi \frac{v \sin \beta}{\lambda}} d v
\end{aligned}
$$

This way, the antiderivatives are defined in the spatial coordinates as:

$$
z(t+1)=\left[H\left(x_{\max }\right)-H\left(x_{\min }\right)\right] e^{-j 2 \pi \frac{R_{0}}{\lambda}}
$$

See now that the integral part in (18b) does not depend on the two-way propagation path between the receiver and the seafloor, i.e. $R_{0}$, but on the spatial position $v$ and arrival angle $\beta$ of the extended scatterers. Actually, we verified that the exponential term in the integral (18b) can be removed provided that the phase variation between two adjacent cells is lower than $2 \pi$. Therefore, the series expansion allows the precalculated grid map, necessary for the computation of the antiderivatives $H(x)$, to be independent on the position of the sonar receiver.

\section{Application to interferometric signals}

In the previous subsections, we verified that the simulated signal held the desired features in terms of cross-correlation between resolution cells in both 1-D and 2-D cases and that the map can be calculated regardless of sensor position. The goal now is to validate that the generated phases interfere, yielding a correct interferometric phase difference. To this end, a 30m-depth flat seafloor was simulated with an obstacle at 100-m across distance, 5-m high and 5-m wide. The simulated seafloor was sampled at $\frac{c \tau}{2 n}$, where $n$ is the number of extended single scatterers per resolution cell, $c$ denotes the in-water sound speed, and $\tau$ is the signal duration. As pointed out in [5], this number of scatterers must be at least equal to three in order to have enough scatterers to generate a correct interferogram.

The generation of Brownian-based interferometric signals can be carried out either through the complete precalculation of the georeferenced grid map defined by (9), or through the series expansion version,

$$
\begin{aligned}
z_{1}(k+1) z_{2}^{*}(k+1)= & {\left[H\left(x_{d}\right)-H\left(x_{c}\right)\right] e^{-j 2 \pi \frac{R_{1}}{\lambda}} } \\
& \times\left[H\left(x_{b}\right)-H\left(x_{a}\right)\right]^{*} e^{j 2 \pi \frac{R_{2}}{\lambda}}(20)
\end{aligned}
$$

The comparison between these two approaches is shown in Figs. 7 and 8, for three and twenty extended single scatterers per resolution cell, respectively. We observe that the seriesbased generated signal (lower plot) contains more "noisy" samples than the complete computation (upper plot) regardless of the number of scatterers per resolution cell. This can be explained by the approximation involved in the Taylor series expansion approach.

\section{E. Discussion}

The Brownian motion approach permits the generation of Gaussian signals at reception that, together with the continuous splitting of the seafloor into extended scatterers, preserves the conservation of energy [5], the right interference between signals (see Figs. 7 and 8), and the decorrelation between different areas of the sea bottom (see Fig. 5). Nevertheless, two main drawbacks limit the implementation of this approach: the first one concerns the georeferenced grid map necessary to compute the Brownian sheet. Indeed, suppose we intend to generate a map, 100-m across-track long and 100-m alongtrack wide, with a sidescan sonar emitting signals of $0.15-\mathrm{ms}$ length. For a three scatterers per resolution cell, the simulator engine should store the position of about seven million scatterers and then precalculate the Brownian sheet before the generation of complex signals. This entails a computational burden in term of time and memory.

The second drawback concerns the inner functioning of the simulator framework [2]. The simulator engine launches 

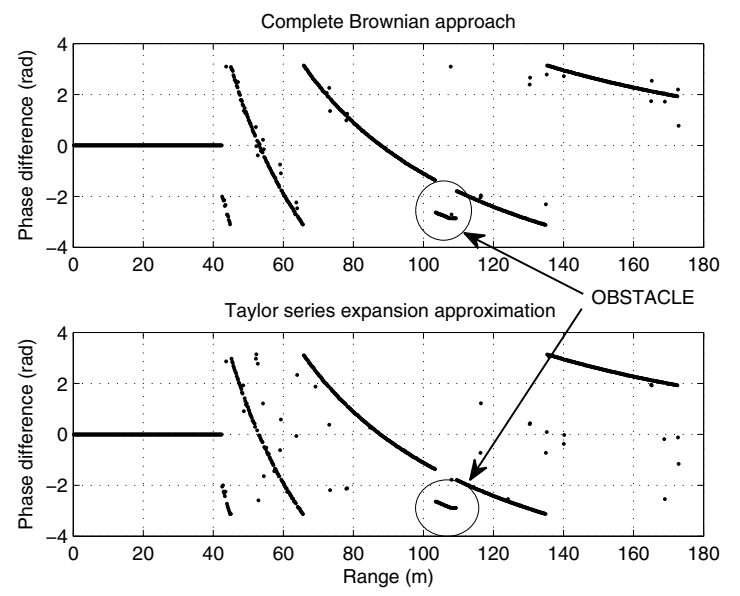

Fig. 7. Comparison of Brownian-based simulated phase differences obtained from a complete computation approach defined by (9) (upper plot), and obtained from the series expansion approach (20) (lower plot) for three extended scatterers per resolution cell. Parameters: $B=4 \lambda, H=30 \mathrm{~m}$, $f=455 \mathrm{kHz}$.

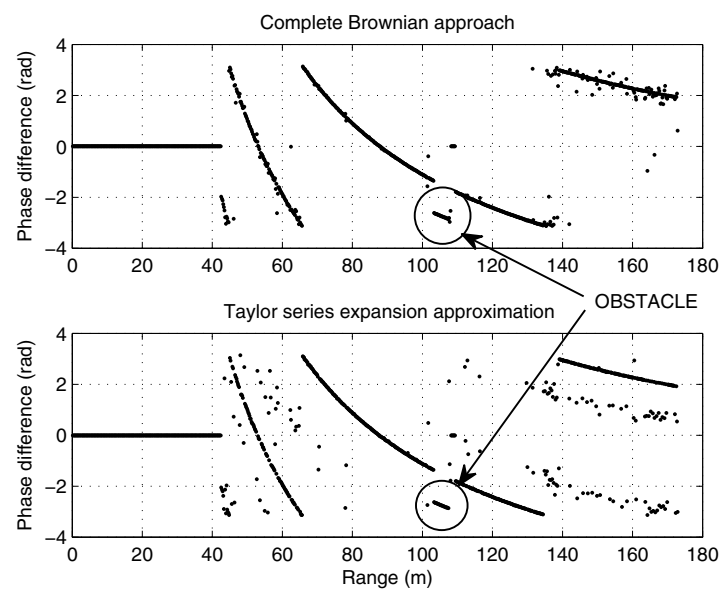

Fig. 8. Comparison of Brownian-based simulated phase differences obtained from a complete computation approach defined by (9) (upper plot), and obtained from the series expansion approach (20) (lower plot) for 20 extended scatterers per resolution cell. Parameters: $B=4 \lambda, H=30 \mathrm{~m}, f=455 \mathrm{kHz}$.

acoustic tubes in order to take into account volume interaction between the acoustic wave and the scene. The intersection of the four rays, delimiting the launched tube, with the scene results in polygons whose form depends on the sea-bottom features. However, so far, the proposed Brownian approach only allows the simulation of rectangular patches and does not permit forms, such as triangles or asymmetric patterns.

\section{CONCLUSIONS AND PERSPECTIVES}

In order to use a simulator for signal or array processing, it is necessary to produce realistic signals with both energy and phase parts matching experimental seafloor characteristics. In this paper, we showed that the contribution of extended scatterers to the received signal can be modeled as an uncorrelated white Gaussian distribution that, after integration of scatterers inside a resolution cell, results in a Brownian motion process. The Gaussian property permits the attribution of a different identity to each scatterer, and thus obtain a spatial decorrelation between signals received from uncorrelated footprints.

Note that in the current implementation, the proposed approach may be computationally memory-consuming because it stores in memory the position of all the scatterers composing the sea-bottom grid map for the current ping, and then, precalculate the Brownian sheet. Moreover, it does not implement polygonal cell footprints, which reduces the application of the tube engine. Finally, the introduction of an attenuation term in the integration of scatterers contribution would compel to precalculate more Brownian maps.

New developments should focus on approaches that assign an identity to extended scatterers independently of its neighborhood, and thus reducing the memory burden. One possibility could be the Perlin noise [16] that could provide a textural information to the signal. However, it may have problems to produce right signal statistics and sensor decorrelation. A more attractive approach would consist in using some extension of the Brownian bridge [19] to enable refinement of an already simulated grid.

\section{REFERENCES}

[1] D. Guériot, S. Daniel, and E. P. Maillard, "Sonar images and swath bathymetry simulations for environment restitution through acoustical ray tracing technique," in Fourth European Conference on Underwater Acoustics, vol. 1, Rome, Italy, Sept. 1998, pp. 425-431.

[2] D. Guériot, C. Sintes, and R. Garello, "Sonar data simulation based on tube tracing," in Oceans 2007 MTS/IEEE Conf. Proc., Aberdeen, Scotland, June 2007, pp. 1-6.

[3] P. D. Mourad and D. R. Jackson, "High frequency sonar equation models for bottom backscatter and forward loss," in Oceans'97 MTS/IEEE Conference Proceedings, Sept. 1989, pp. 1168-1175.

[4] E. Y. T. Kuo, "Wave scattering and transmission at irregular surfaces," The Journal of the Acoustical Society of America, vol. 36, no. 11, pp. 2135-2142, 1964.

[5] C. Sintes and D. Guériot, "Interferometric sonar signal simulation through tube tracing," in Oceans 2008 MTS/IEEE Conf. Proc., Kobe, Japan, Apr. 2008, pp. 1-8.

[6] W. S. Burdic, Underwater Sound System Analysis. Englewood Cliff: Prentice-Hall, 1984.

[7] C. de Moustier, "State fo the art in swath bathymetry survey systems," in Int. Hydr. Rev., 1988, vol. 64, pp. 25-54.

[8] P. N. Denbigh, "Swath bathymetry: Principles of operation and an analysis of errors," IEEE J. Oceanic Eng., vol. 14, no. 4, pp. 289-298, Oct. 1989.

[9] X. Lurton, "Swath bathymetry using phase difference: theoretical analysis of acoustical measurement precision," IEEE J. Oceanic Eng., vol. 25, no. 3, pp. 351-363, July 2000.

[10] G. Llort-Pujol, C. Sintes, and X. Lurton, "A new approach for fast and high-resolution interferometric bathymetry," in Oceans 2006 - Asia, Singapore, May 2006, pp. 1-7.

[11] C. Oliver and S. Quegan, Understanding synthetic aperture radar images. Boston: Artech House, 1998.

[12] N. Levanon, Radar principles. New York: Wiley-Interscience, 1998.

[13] G. Jin and D. Tang, "Uncertainties of differential phase estimation a associated with interferometric sonars," IEEE J. Oceanic Eng., vol. 21, no. 1, pp. 53 - 63, Jan. 1996.

[14] C. Sintes, G. Llort-Pujol, and D. Guériot, "Coherent probabilistic error model for interferometric sidescan sonars," IEEE J. Oceanic Eng., to be published.

[15] R. J. A. Tough, D. Blacknell, and S. Quegan, "A statistical description of polarimetric and interferometric synthetic aperture radar data," Proc. Royal Society, vol. 449, pp. 567-589, 1995. 
[16] K. Perlin, "An image synthesizer," in Computer Graphics magazine, 1985, vol. 19 , no. 3 .

[17] B. Pesquet-Popescu and J. L. Vehel, "Stochastic fractal models for image processing," in IEEE Signal Processing Magazine, 2002, vol. 19, no. 5, pp. 48-62.

[18] D. Saupe, Algorithms for random fractals, ser. The science of fractal images. New York: Springer-Verlag, 1988, ch. 2, p. 71136.

[19] D. Khoshnevisan and Y. Xiao, "Images of the brownian sheet," in Trans. Amer. Math. Soc., 2007, vol. 359, pp. 3125-3151. 